Annuaire suisse de politique de développement

14 | 1995

Annuaire Suisse - Tiers Monde 1995

\title{
Pour une politique suisse plus cohérente envers les pays du Sud
}

Quelques réflexion critiques à partir du cas de l'Indonésie

Jean-Luc Maurer

\section{CpenEdition}

\section{Journals}

Édition électronique

URL : http://journals.openedition.org/aspd/1447

DOI : $10.4000 /$ aspd. 1447

ISSN : 1663-9669

\section{Éditeur}

Institut de hautes études internationales et du développement

Édition imprimée

Date de publication : 1 février 1995

Pagination : 164-173

ISSN : $1660-5934$

\section{Référence électronique}

Jean-Luc Maurer, "Pour une politique suisse plus cohérente envers les pays du Sud », Annuaire suisse de politique de développement [En ligne], 14 | 1995, mis en ligne le 03 mai 2013, consulté le 08 septembre 2020. URL : http://journals.openedition.org/aspd/1447 ; DOI : https://doi.org/10.4000/ aspd. 1447 


\title{
Pour une politique suisse plus cohérente enver les pays du Sud
}

\section{Quelques réflexion critiques à partir du cas de l'Indonésie}

\author{
Jean-Luc Maurer, Directeur IUED
}

L'Indonésie est une excellente étude de cas pour mener une réflexion sur la cohérence de la politique extérieure de la Suisse à l'égard des pays du Sud. Tout d'abord, c'est - derrière la Chine et l'Inde - le troisième plus grand des pays en développement de la planète et il est le seul en Asie du Sud-Est sur lequel la Confédération a décidé depuis le début des années 70 de concentrer son aide publique. De plus, c'est un pays qui restera encore longtemps confronté à de graves problèmes de développement, mais qui commence à émerger comme partenaire économique et commercial significatif sur la scène 
régionale et internationale, et où les intérêts privés helvétiques sont anciens et non négligeables. Finalement, c'est un pays dont la politique de développement a donné de remarquables résultats économiques et sociaux, mais qui est aussi caractérisé par un régime autoritaire ayant une conception très particulière du respect des droits de l'homme et où la pression excercée sur les ressources naturelles pose de graves problèmes d'environnement. Bref, c'est donc à priori un partenaire idéal pour penser et essayer de mettre en oeuvre une politique extérieure plus cohérente. Avant d'examiner ce que devraient être les principaux objectifs et ce qui pourrait constituer le mécanisme central d'une telle politique, passons en revue les quatre niveaux de contradictions possibles en termes de cohérence.

\section{Les relations Suisse-Indonésie: quatre niveaux d'analyse possibles}

\section{Coopération au développement et développement économique}

La Suisse a été très active dans le développement de l'Indonésie depuis le début des années 70 , quand le nouveau gouvernement issu des événements politiques sanglants de 1965-66 a fait appel à l'aide occidentale pour financer le relèvement économique de l'archipel et que ce dernier est devenu le pays de concentration de la DDA en Asie du Sud-Est. En dehors du fait qu'elle fait partie du consortium d'aide à l'Indonésie (IGGI devenu CGI) et contribue chaque année au financement multilatéral de son développement, la Suisse a réalisé en 25 ans un programme de coopération bilatéral certes modeste, mais bien conçu et apprécié du partenaire indonésien pour son esprit et sa qualité. Ce programme a mis l'accent sur la formation technique professionnelle et l'amélioration des conditions de vie des populations défavorisées, plutôt en milieu urbain à Java et largement en milieu rural dans les autres îles.

Sans entrer ici dans les détails ${ }^{1}$, I'Indonésie a fait pendant ce quart de siècle des progrès immenses au niveau de son développement. Considérée par certains au début des années 60 comme le cas de sous-développement le plus flagrant de la planète, elle apparaît aujourd'hui à beaucoup comme l'un des prochains NPI d'Asie. Ce succès fulgurant est tout d'abord le fruit d'une politique de développement pragmatique et judicieuse de la part du gouvernement indonésien, qui a su établir les bonnes priorités et réaliser les investissements nécessaires. Mais il doit aussi beaucoup au soutien que cette politique a obtenue de l'extérieur de la part de la Banque mondiale, du Japon et des pays occidentaux regroupés au sein de I'IGGI. Dans ce cadre, le rôle de la Suisse, bien que limité, n'a pas été négligeable. En d'autres termes, nous avons contribué à la réalisation d'une "histoire de développement à succès" dans un grand pays du Sud. Le cas est suffisamment rare depuis ie début des

Voir à ce sujet le chapitre de l'auteur sur le développement économique, social et politique de I'Indonésie dans l'ouvrage de G. Etienne, J.-L. Maurer et C. Renaudin, Suisse-Asie. pour un nouveau partenariat, Genève, Editions Olizane et CRAM (IUHEI/IUED), 1992, pp. 83-128. 
indépendances dans le Tiers Monde pour être souligné avec force. On devrait d'ailleurs s'en féliciter ouvertement et le faire savoir beaucoup plus largement auprès d'une opinion publique que les media ont plutôt tendance à sensibiliser sur l'échec de la coopération internationale en Afrique.

Au cours de ce processus de développement, le PNB indonésien par tête a été multiplié par plus de onze, passant de US\$60 en 1969 à 670 en 1992. Le bond en avant est certes impressionnant, mais le résultat atteint est encore bien modeste puisque l'Indonésie est, selon la Banque mondiale, tout juste à la limite entre les économies à faible revenu et à revenu intermédiaire, au même niveau que des pays dont on pourrait à priori penser qu'ils sont nettement moins développés qu'elle comme l'Egypte, la Côte d'Ivoire ou la Bolivie, et largement en dessous du Pérou par exemple, qui atteignait déjà quasiment la barre des US\$1000 en 1992. C'est pourtant bien en prétextant du fait que I'Indonésie s'était fortement développée depuis vingt ans et ne correspondait plus aux principaux critères de la Loi sur la coopération au développement de 1976 - précisant que notre APD doit se concentrer sur les pays, les régions ou les groupes de population les plus pauvres - que la DDA a décidé de se retirer de ce pays à l'horizon 2000. Or, selon les sources les plus sérieuses, 15 à $20 \%$ de la population indonésienne, soit 30 à 35 millions de personnes, vivent encore en dessous de la ligne de pauvreté absolue, la plupart d'entre eux à Java, l'île la plus développée mais aussi la plus peuplée de l'archipel. Ce seul chiffre parle de lui-même.

En partant de cette analyse toute simple, et puisque ce dossier de l'ASTM a pour objet la réflexion sur la cohérence de notre politique extérieure, qu'il nous soit permis de dire que cette décision de retrait ne nous semble pas en avoir constitué la meilleure démonstration. Elle a d'ailleurs été prise sans grande consultation ni débat, presque en catimini, avec une hâte un peu honteuse. C'est fort dommage, car si la réflexion sur la cohérence que nous prônons depuis des années à propos de l'Indonésie avait eu lieu dans la concertation, elle aurait peut-être débouché sur une décision différente qui offrirait aujourd'hui un éventail de possibilités plus large pour notre politique extérieure à l'égard de cette importante puissance régionale d'Asie. Par ailleurs, même si la décision de se retirer avait au bout du compte primé, la transmission du relais à d'autres partenaires nationaux aurait pu se faire dans de meilleures conditions que celles qui consistent à laisser tomber le témoin en espérant qu'il va être ramassé. Enfin, même si un tel processus de consultation avait débouché sur une décision de retrait, il aurait été possible de faire savoir haut et fort à l'opinion publique suisse que la DDA se retirait d'Indonésie pour cause de succès probant en matière de développement et qu'elle passait à ce stade le relais à d'autres acteurs nationaux plus directement concernés.

\section{Coopération au développement et relations économiques extérieures}

Depuis vingt ans, en se développant, l'Indonésie est aussi devenue un partenaire commercial et économique de plus en plus important pour la 
Suisse. Certes, les grandes entreprises multinationales helvétiques comme Nestlé, Ciba Geigy, Diethelm ou la SGS y sont implantées depuis longtemps, conscientes de l'énorme potentiel de l'économie indonésienne et d'un marché domestique de bientôt 200 millions d'habitants. Toutefois, surtout depuis la libéralisation économique des dix dernières années, les échanges et les investissemenss entre les deux pays se sont accrus. L'Indonésie en arrive à un stade de son développement industriel où elle a besoin de passer les paliers technologiques qu'ont franchis avant elle la Corée du Sud, Taiwan, Hong Kong, Singapour ou la Thailande et la Malaisie. C'est à ce stade que le savoir-faire de nombreuses petites et moyennes industries helvétiques devrait pouvoir lui être le plus utile. Nos entreprises de services sont aussi assez bien placées en la matière, tant dans le domaine des banques et des assurances que de l'hôtellerie et du tourisme. Les perspectives économiques sont donc à priori plutôt bonnes, pour autant que ces entreprises soient prêtes à prendre des risques. II semble que se soit malheureusement là une autre paire de manches. En tous les cas, les milieux d'affaires de la plupart des autres pays industrialisés ont bien compris les enjeux et sont en moyenne plus actifs que les nôtres sur ce marché porteur.

D'un autre côté, l'Indonésie restera encore pour longtemps un pays en développement, avec ses classiques problèmes économiques et sociaux. De ce fait, elle demeurera pendant une bonne partie de la première moitié du siècle prochain éligible au titre de l'aide publique au développement du Japon, son premier partenaire économique et bailleur de fonds, ainsi que de la plupart des grands pays occidentaux qui y sont très impliqués comme l'Allemagne, l'Australie ou les Etats-Unis. La majorité de ces derniers continueront donc à mener vis-à-vis de l'Indonésie une politique extérieure correspondant à la nature "dualiste" de ce pays, à la fois récipiendaire de ressources provenant de la coopération au développement et partenaire économique et commercial à part entière. C'est la seule politique judicieuse à suivre vis-à-vis de tels pays en développement, se situant dans une phase de transition entre une économie encore largement traditionnelle et la société industrielle de consommation.

Le problème est que notre système administratif fédéral est peu propice à ce genre de politique. La coopération au développement est l'affaire du DFAE et de la DDA alors que les relations économiques et commerciales sont celle du DFEP et de l'OFAEE. En conséquence, soit un pays est pauvre comme le Népal, et la DDA en a l'exclusivité, soit il est devenu plus aisé comme la Corée et il tombe sous la coupe de l'OFAEE. Bref, c'est plutôt le règne du cou bien - ou bien" typiquement helvétique que celui d'une politique plus subtile et nuancée que nécessiteraient la plupart des situations. Or, entre le "trade not aid" des émules de l'ultralibéralisme et le "aid without trade" de certains intégristes de la coopération, il y a un juste milieu à trouver, en particulier pour des pays en transition de ce type. Ce qui est peut-être valable pour le Niger ou le Népal ne l'est en tout cas pas du tout pour l'Indonésie, et bientôt probablement plus pour l'Inde et le Pakistan, les deux principaux pays de concentration de la DDA dans le sous-continent. 
Par ailleurs, la collaboration entre la DDA et l'OFAEE n'a jamais été très étroite ni même vraiment bonne. II y a plus de rivalité et de méfiance entre les fonctionnaires des deux administrations que de volonté de collaborer à l'élaboration d'une politique cohérente concertée. C'est là que réside notre contradiction majeure. A-t-on réellement la volonté et la capacité d'élaborer une politique économique extérieure cohérente avec les pays du Sud - notamment ceux de nature "dualiste" comme l'Indonésie - qui permette à la fois de continuer à mener des activités de coopération publique au développement et d'augmenter le volume des relations commerciales et des investissements privés? Comment faire, maintenant que la décision d'interrompre le programme de coopération en Indonésie a été prise, pour rattraper ce qui peut encore l'être en la matière? Le rythme très rapide du désengagement ne facilite en tous les cas pas les choses. Sans vouloir trop insister, il nous semble que l'on a quand même déjà largement raté là l'occasion de mettre sur pied une politique vraiment cohérente à l'égard d'un pays du Sud, alors que le cas de l'Indonésie eut une nouvelle fois été idéal pour le faire.

\section{Développement économique et démocratisation du système politique}

En dehors d'un développement trop rapide qui lui pose paradoxalement le genre de problèmes susmentionnés, l'autre difficulté majeure à laquelle l'Indonésie a toujours eu à faire face en Suisse, et dans beaucoup d'autres pays occidentaux, est liée à la nature autoritaire et répressive du régime militarotechnocrate issu des terribles événements sanglants de 1965-66. S'il a indubitablement sorti le pays du marasme économique et de la spirale de la paupérisation dans lesquels il était plongé, l'Ordre Nouveau du Général Suharto qui règne à Jakarta depuis lors est en effet loin de respecter les règles démocratiques les plus élémentaires.

Le problème le plus grave est lié à l'annexion du Timor Oriental en 1975 et de la répression politique qui y règne depuis, malgré les efforts louables de développement économique qui y sont fait. Mais le cas n'est pas unique et on trouve des problèmes de répression systématique du droit des peuples à disposer d'eux-mêmes ailleurs dans l'archipel. Par ailleurs, les libertés syndicales sont encore largement foulées au pied: le syndicat officiel est à la botte du pouvoir et les velléités d'expression autonome moins complaisante sont violemment réprimées, ainsi que le démontrent certains événements récents des plus tragiques. Enfin la liberté d'expression et de la presse sont étroitement contrôlées, les incessants problèmes auxquels ont à faire face les associations d'étudiants ou la fermeture récente de trois des principaux hebdomadaires nationaux étant là pour le prouver. En bref, l'Ordre Nouveau indonésien est une démocratie dirigée et partielle, qui respecte rituellement les apparences de la consultation électorale quinquennale, mais qui est menée avec une réelle main de fer, même si le gant reste de velours.

Bien que ces aspects n'aient apparemment pas joué de rôle formel dans la décision de la DDA d'interrompre son programme d'ici l'an 2000, il est certain 
que l'image de l'Indonésie n'a jamais été bonne auprès de l'opinion publique occidentale, surtout après les événements sanglants de fin 1991 à Timor. Cela se comprend aisément. Toutefois ne peut-on pas aussi voir le problème sous un autre angle? A notre connaissance, jamais ni nulle part la démocratie n'a précédé le développement. Sans remonter à l'Antiquité, les peuples se sont d'abord occupés d'avoir "le ventre plein" avant de revendiquer des libertés moins matérielles. L'histoire européenne depuis le Moyen Age le démontre amplement. Plus récemment, ce sont aussi des régimes autoritaires et plutôt répressifs très semblables à celui de l'Indonésie du Général Suharto qui ont permis le fulgurant développement de la Corée du Sud ou de Taiwan. Ce processus de développement a donné naissance à de nouveaux acteurs sociaux, une classe ouvrière de plus en plus consciente de sa force qui s'est organisée, et une classe moyenne n'acceptant plus d'être laissée à l'écart du pouvoir politique. Depuis quelques années, ces régimes autoritaires ont commencé à se démocratiser et doivent faire face à des revendications croissantes pour plus de libertés individuelles et collectives.

De fait, les régimes autoritaires développeurs sont toujours victimes de leur propre succès. L'Indonésie ne fait pas exception à la règle, mais avec le temps de décalage qui est lié à son niveau de développement plus bas. La classe ouvrière a commencé à s'organiser et la classe moyenne à revendiquer. II faut aussi dire à la décharge de l'Indonésie, le plus grand pays musulman du monde, qu'il y règne une liberté confessionnelle, un respect de la diversité culturelle et une tolérance générale qui deviennent de plus en plus rares, tant dans les pays en développement du Sud que dans les pays du Nord que l'on pensait être développés. Est-il besoin à cet égard de rappeler le cas de la Yougoslavie? La poursuite du développement ne peut que contribuer à l'accélération de ce processus. Ainsi, plutôt que de faire au gouvernement indonésien une morale hypocrite d'inspiration judéo-chrétienne sur les droits de l'homme et la démocratie - attitude largement déplacée compte tenu des performances discutables de l'Occident en la matière et fort mal reçue étant donné l'expérience historique traumatisante vécue du temps de la colonisation -, ne vaudrait-il pas mieux continuer à soutenir un processus de développement qui a toutes les chances de produire rapidement les effets sociaux et politiques escomptés?

Par ailleurs, on peut aussi se dire que ve n'est pas en partant et en s'abstenant que l'on a le plus de chances de pouvoir peser sur les orientations politiques d'un gouvernement. Au contraire, c'est seulement en s'impliquant plus au niveau de son développement et en ayant des exigences croissantes en tant que partenaire à part entière que l'on peut approfondir le dialogue. Ce dernier ne peut en effet se fonder que sur des relations de confiance. Or la confiance ne se gagne pas en un jour. Toute l'énergie dépensée depuis vingt-cinq ans en Indonésie pour établir une telle relation, mieux connaître ce pays complexe et apprendre à travailler avec un partenaire difficile est largement hypothéquée par la décision d'un retrait si précipité. II nous faudra maintenant partir de zéro avec un autre pays de concentration en Asie 
du Sud-Est, le Vietnam, où presque tout le monde se précipite d'ailleurs en même temps. II s'agit certes d'un pays dont les besoins sont immenses mais sur lequel le niveau de connaissances accumulées en Suisse est encore bien faible, où les problèmes économiques et sociaux sont à peu près de même nature qu'en Indonésie, où le régime politique est au moins aussi autoritaire, où la corruption n'est guère moins répandue et où la situation des droits de l'homme n'est en tous les cas pas plus brillante.

\section{Développement économique rapide et atteintes à l'environnement}

Si l'on considère que les problèmes d'environnement découlent largement de la surpopulation, il faut également reconnaître au régime indonésien de grands mérites dans ce domaine. La politique de limitation des naissances qu'il a mise en place depuis une vingtaine d'années a en effet montré des résultats très encourageants, puisque le taux de croissance naturelle est tombé sous la barre des $2 \%$. En revanche de nombreux autres problèmes d'environnement se posent. Le plus grave est celui de la déforestation galopante des forêts tropicales de Bornéo et d'autres îles. Toutefois, là aussi, la solution n'est pas dans un discours moralisateur sur la gestion rationnelle des ressources forestières en tant que patrimoine commun de l'humanité. L'Occident est mal venu pour venir faire la morale à ce sujet, après avoir dévasté la planète et exterminé les peuples indigènes quel que soit l'endroit où il s'est rendu pour y imposer sa domination. La solution réside plutôt dans le soutien à apporter à la formation d'une conscience environnementale indonésienne qui émerge face aux abus d'un développement trop axé sur le profit économique immédiat et les valeurs purement matérielles. D'autres problèmes d'environnement substantiels se posent au niveau de la gestion de l'eau, de l'usage abusif des pesticides, de l'extraction minière ou de la pêche hauturière. On ne pourrait cependant pas mieux contribuer à leur résolution qu'en soutenant les ONG locales les plus actives dans ce secteur.

\section{Priorisation des objectifs et recherche d'une meilleure cohérence}

\section{Objectif central: soutenir le processus de développement indonésien}

L'objectif global de la politique extérieure suisse à l'égard de l'Indonésie devrait logiquement être que le niveau de vie de la population continue à augmenter, que la démocratisation du système politique progresse et que les problèmes d'environnement trouvent leurs solutions. Pour ce faire, il faut que le pays poursuive son développement, en diversifiant son économie et en accélérant son industrialisation, afin de créer des emplois et de pouvoir absorber les énormes cohortes de jeunes arrivant sur le marché du travail chaque année. A cet effet, il a besoin de former un nombre croissant de jeunes Indonésiens qualifiés pour le marché du travail. Cela signifie que les champs de l'éducation et de la formation professionnelle - dans lesquels la Coopéra- 
tion suisse au développement s'est fortement impliquée depuis 25 ans - restent prioritaires. Or, la poursuite d'un appui extérieur octroyé au titre de l'aide publique au développement demeure vraisemblablement nécessaire à ce stade pour que les réformes importantes entreprises soient réellement menées à leur terme. C'est bien ce qu'a compris un pays comme l'Allemagne, qui a décidé de mettre dans la balance tout son poids et sa longue expérience dans ce secteur.

La priorité reste donc à l'amélioration de la situation économique, condition de base pour continuer à faire diminuer la croissance démographique, à améliorer le niveau de vie général de la population et à réduire les écarts de revenus entre les catégories sociales, à favoriser l'émergence des nouveaux acteurs sociaux qui exerceront des pressions croissantes pour une plus grande démocratisation du système politique. Bref il faut continuer à aider ce pays à se développer économiquement car c'est le seul moyen pour que les autres problèmes évoqués plus haut au niveau social et politique trouvent progressivement une solution.

En conséquence, et au titre de la cohérence de sa politique extérieure, il serait logique que la Suisse continue à soutenir le développement économique de l'Indonésie, tant à travers son aide publique que par l'intensification de ses échanges commerciaux avec ce pays et de ses investissements privés sur place. Idéalement, cela impliquerait que la décision de mettre un terme au programme de coopération avec l'Indonésie d'ici l'an 2000 soit réexaminée et que le rythme du désengagement soit au moins revu à la baisse. II serait alors certainement plus facile de mettre en place et de rôder les mécanismes de collaboration à même d'assurer que la DDA puisse passer le témoin des relations économiques bilatérales à d'autres acteurs nationaux publics comme I'OFAEE ou l'OSEC et à des acteurs privés comme la nouvelle chambre de commerce Suisse-Asie du Sud-Est ou les associations professionnelles des branches industrielles concernées ainsi que les organisations non gouvernementales ou confessionnelles qui souhaitent continuer à participer directement au développement économique et social indonésien. Cela dit, les échéances très rapprochées qui ont été fixées pour le désengagement peuvent aussi constituer un facteur positif, en obligeant les acteurs concernés à agir rapidement sous la contrainte. Quoi qu'il en soit, tout cela implique aussi que, parallèlement, les sphères privées helvétiques, en particulier les PME, sortent de leur réserve et s'intéressent plus activement à l'énorme potentiel de ce vaste pays.

D'un autre côté, cela ne devrait pas remettre en cause la décision prise par la DDA de développer progressivement un programme de coopération avec le Vietnam comme nouveau pays de concentration en Asie du Sud-Est. En fait, un désengagement progressif et sensiblement plus lent que prévu en Indonésie pourrait être couplé avec la montée en puissance du Vietnam. Or, il faudra bien 10 à 15 ans pour développer un programme de coopération avec le Vietnam comparable à celui qui a été mis sur pied avec l'Indonésie. En tout premier lieu, il faudra d'abord faire ce qui est nécessaire pour former en Suisse un pool de compétences comparable à celui dont nous disposons sur 
l'Indonésie, indispensable pour travailler efficacement dans ce nouveau pays. Par ailleurs, si le Vietnam se développe au rythme où il prétend le faire, il n'est pas impossible qu'il atteigne un niveau de développement comparable à celui de l'Indonésie d'aujourd'hui avant que le programme de la DDA n'y soit vraiment significatif. C'est la raison pour laquelle il faudrait mettre en place d'entrée de jeu les mécanismes de coordination à l'échelle nationale qui permettront de s'en retirer dans de meilleures conditions que cela ne risque d'être le cas en Indonésie, quand ce pays aura lui aussi atteint, et peut-être plus vite que prévu, un niveau de développement qui le rendra inéligible au titre de l'APD suisse.

\section{Favoriser la cohérence à travers la coordination triangulaire}

II est certain que la Loi sur la Coopération au développement de 1976 n'est à priori pas très adaptée à l'élaboration de politiques extérieures cohérentes visà-vis de pays en développement engagés dans une transition économique comme l'Indonésie. Toutefois, il faut éviter dans le contexte actuel de la remettre en question et même de l'amender. Le danger est trop grand "d'ouvrir la boîte de Pandore". En revanche il est indispensable d'élaborer les critères qui permettent d'avoir, au niveau des modalités d'application de la loi, une interprétation beaucoup plus flexible en fonction du type de pays en développement concerné.

Plus fondamentalement, il nous semble que la base de toute politique cohérente future avec l'Indonésie, ou tout autre pays en développement entrant dans la même catégorie, passe par la mise en place de mécanismes de coordination et de collaboration entre l'administration publique, les milieux de l'économie privée et le monde universitaire. Pour l'instant, la situation à cet égard est particulièrement malsaine en Suisse. En effet, l'administration publique travaille de son côté, sans beaucoup de relations avec le secteur privé et les universités: le secteur privé regarde habituellement de haut l'administration publique et le monde universitaire alors que ce dernier est à la fois frustré de ne pas être consulté et largement incapable de sortir de sa tour d'ivoire et de se remettre en cause de manière tant soit peu sérieuse. Face à cela, il s'agit en fait d'établir entre ces trois pôles la fameuse "triangulation" que certains pays pratiquent déjà depuis longtemps, le Japon avec un art consommé et inégalé, d'autres comme les USA ou la RFA en tous cas mieux que la Suisse.

Tout d'abord, avant que la coordination puisse se faire entre les trois partenaires concernés, il est nécessaire que chacun d'eux se renforce. Du côté de l'administration publique, un travail essentiel doit ainsi être fait entre la DDA et l'OFAEE qui doivent apprendre à mieux se connaître et à travailler ensemble. D'autres offices fédéraux sont également concernés. Du côté des milieux de l'industrie privée, il est indispensable que les grandes entreprises intensifient leurs relations avec les PME dans chaque branche d'activité et jouent un rôle de parrainage à leur égard. II est par ailleurs également vital 
que le patronat associe beaucoup plus les syndicats à l'élaboration des politiques industrielles vis-à-vis des pays du Sud. II faut enfin que les ONG apprennent ' sollaborer, plutôt que de s'ignorer ou d'être en perpétuelle compétition. Du culé du monde universitaire, les différents centres d'excellence du pays devraient apprendre à jouer sur leurs complémentarités et à partager leurs expériences. Cela étant fait, il faudrait alors que les trois pôles du triangle établissent des inter-relations et les renforcent. Ainsi l'administration publique doit s'habituer à travailler plus étroitement avec les milieux de l'industrie privée et ces derniers à la dénigrer un peu moins, alors que le monde universitaire doit apprendre à mieux collaborer avec ces deux partenaires, en développant avec eux des relations plus amples. Tout cela implique donc que les trois acteurs concernés soient prêts à se remettre en question et fassent preuve d'esprit de conciliation.

Le processus de triangulation susmentionné est complexe et prendra du temps. Afin d'aller de l'avant sans tarder, la meilleure chose est d'apprendre en pratiquant. Le cas de I'Indonésie se prête bien à un tel exercice. On pourrait donc imaginer par exemple un première expérience de triangulation qui se manifesterait par la mise en place d'une commission nationale tripartite sur l'Indonésie. Elle serait chargée d'examiner tous les éléments de politique émanant des trois partenaires, d'en assurer la coordination et d'en éliminer les contradictions majeures afin d'établir un programme intégré et harmonieux qui permette à la Suisse de mener une politique extérieure cohérente à l'égard de ce pays. Le même excercice devrait être fait pour tous les grands pays en développement de nature comparable avec lesquels la Suisse entretient des relations de coopération, des échanges commerciaux et des collaborations universitaires. En plus de l'Indonésie, on pense en tout premier lieu à la Chine, à l'Inde, au Pakistan et au Vietnam en Asie, au Brésil, au Mexique, au Chili, à l'Argentine et au Pérou pour l'Amérique latine, ainsi qu'à l'Afrique du Sud. Quand chacune de ces commissions nationales tripartites fonctionnera et aura acquis un minimum d'expérience, on pourra alors penser à établir une commission mixte tripartite pour chaque pays, comprenant également des représentants des trois partenaires du côté du pays du Sud.

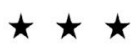

Commençons toutefois par le commencement, c'est-à-dire par l'Indonésie. Le moment est bien choisi pour le faire et peut-être pourrons-nous élaborer ainsi une politique extérieure plus cohérente par laquelle la DDA passera progressivement le témoin à l'OFAEE, aux ONG, à l'économie privée et aux universités concernées d'ici la fin du siècle. Formons donc cette commission nationale tripartite sur l'Indonésie sans tarder. Ensuite, tâchons de faire tout de suite de même pour le Vietnam, afin de partir d'un meilleur pied dans la coopération qui s'esquisse avec ce pays. 